Revue d'histoire de l'Amérique française

ZWB REVUE D.HISTOIRE DE L'AMÉRIQUE FRANÇAISE

\title{
La « Farmer's Bank of Rustico » - Une des premières banques du peuple
}

\section{John T. Croteau}

Volume 10, numéro 1, juin 1956

URI : https://id.erudit.org/iderudit/301741ar

DOI : https://doi.org/10.7202/301741ar

Aller au sommaire du numéro

Éditeur(s)

Institut d'histoire de l'Amérique française

ISSN

0035-2357 (imprimé)

1492-1383 (numérique)

Découvrir la revue

Citer cet article

Croteau, J. T. (1956). La "Farmer's Bank of Rustico " - Une des premières banques du peuple. Revue d'histoire de l'Amérique française, 10(1), 13-48. https://doi.org/10.7202/301741ar d'utilisation que vous pouvez consulter en ligne.

https://apropos.erudit.org/fr/usagers/politique-dutilisation/ 


\section{LA «FARMERS' BANK OF RUSTICO 》 - UNE DES PREMIEERES BANQUES DU PEUPLE}

\section{(HISTOIRE ÉCONOMIQUE)}

La «Farmers' Bank of Rustico», établissement bancaire organisé par les cultivateurs acadiens de l'île du Prince-Édouard, a obtenu une charte de la législature coloniale et a pratiqué ses opérations de 1864 à 1894. Pour plusieurs raisons, cette banque présente un intérêt historique; elle fut la première banque du peuple fondée au Canada - et peut-être en Amérique du Nord; elle est sans contredit la plus petite banque à charte, quant au capital-actions, qui ait jamais fait affaires au Canada - et peutêtre dans l'Empire Britannique; elle a prouvé qu'une institution de crédit peut effectivement aider à faire prospérer une population appauvrie; et, ce qui est peut-être plus important, elle a inspiré Alphonse Desjardins, le premier organisateur des caisses populaires en Amérique du Nord, et ainsi elle est devenue le précurseur du mouvement des caisses populaires sur ce continent.

Cette étude traitera d'abord du passé historique des cultivateurs acadiens qui ont fondé cette banque. Elle portera également sur la monnaie, les opérations bancaires et le crédit, tels qu'ils existaient dans l'île du Prince-Édouard vers le milieu du dix-neuvième siècle. Seront expliquées les grandes lignes de la loi constituant en corporation la «Farmers' Bank of Rustico », ainsi que les difficultés qui en ont précédé l'adoption par la reine Victoria. Après l'entrée de l'île du Prince-Édouard dans la Confédération, en 1873, cette petite banque était, en pratique, condamnée à disparaître; cependant, elle a continué à pratiquer des opérations profitables durant vingt années.

Les opérations financières de la Banque de Rustico seront exposées dans plusieurs de ses bilans. On expliquera ce qui est survenu lorsque la Banque a cessé ses opérations, ainsi que l'in- 
fluence qu'elle a exercée dans l'élaboration du système d'Alphonse Desjardins. Et, en dernier lieu, on estimera dans quelle mesure cette banque a inspiré les opérations des banques du peuple.

La portée des opérations et la structure des banques du peuple ont grandement varié dans divers pays, selon les institutions juridiques auxquelles ces banques ont dû se conformer. ${ }^{1}$ Les techniques d'opération, telles que le droit de vote, la qualité de membres et l'organisation des comités, diffèrent également dans une large mesure. Cependant, les banques du peuple présentent ordinairement plusieurs traits communs: 1. propriété dévolue $\mathrm{au}$ « peuple» (i.e. les classes à faible revenu, comprenant ouvriers ou cultivateurs) ; 2 . gestion active des propriétaires par l'entremise de fonctionnaires élus, et non abdication du contrôle entre les mains de gérants professionnels; 3 . constitution en corporation avec actions de valeur peu élevée, afin de permettre aux personnes touchant un revenu minime de devenir propriétaires; 4. ajustement des opérations aux besoins des membres - les heures d'affaires, ainsi que le montant et les conditions des emprunts, sont fixées de façon à accommoder les emprunteurs; 5 . locaux modestes et gestion économique, ou encore «frugale»; 6. ordinairement, une banque du peuple dessert une classe dont l'occupation, la religion et l'origine ethnique sont semblables; et 7. le principe qui l'inspire est de servir, et non de spéculer.

D'après ces critères, la « Farmers' Bank of Rustico » semblerait avoir été la première vraie banque du peuple au Canada. Bien que la désignation «Farmers » ou «Agricultural » apparaisse dans le titre de plusieurs banques canadiennes vers les années 1830, ces banques étaient des entreprises privées fondées avant 1841, alors qu'a été requise l'obtention d'une charte. L'appellation n'avait d'autre but que d'inviter le cultivateur à faire affaires avec cette banque. Les premiers projets de banque ont tous été tentés dans les cités et villes; ces banques étaient con-

1 Donald S. Tucker, The Evolution of People's Banks (New-York: Longmans, Green and Co., 1922), 242 et suiv. 
trôlées par les classes commerciales bien que, à cette époque, la grande majorité de la population fût rurale; et l'on peut donc en conclure que les cultivateurs formaient une grande partie de la clientèle des banques. ${ }^{2}$

Il n'est pas étonnant que la première banque du peuple ait surgi chez un groupement d'Acadiens. Leur passé les inclinait à se grouper en une communauté étroite. On pouvait s'y attendre: ils ne manqueraient pas de se donner des institutions économiques fondées sur la solidarité collective. «Evangéline », le grand poème de Longfellow, a popularisé l'histoire des Acadiens qui ont été expulsés de la Nouvelle-Écosse en 1755. Cependant, on connaît moins la déportation, en 1758 , des Acadiens de l'île du Prince-Édouard, qui s'appelait alors l'Île Saint-Jean. En 1758, 4,000 à 5,000 colons acadiens établis sur l'Île du Prince-Édouard

2 C. S. Howard, Canadian Banks and Bank-Notes - Données reproduites du Canadian Banker, Vol. 57, no 1 (Winter, 1950) : 30-66. L'étude de Howard contient une liste complète, fondée sur les recherches initiales de H. C. McLeod et complétées par Howard, de toutes les banques canadiennes, indiquant la date de leur établissement et de la cessation de leurs affaires. Dans cette liste figurent quatre banques existant avant 1864 et dont la désignation fait mention des mots «agricultural 》, «farmers 》 ou «people». La «Agricultural Bank of Upper Canada 》, 1834-1838, était une petite institution établie en 1835; elle a fait misérablement faillite en 1837. Les promoteurs ou administrateurs se sont enfuis, et il est indubitable qu'il $\mathrm{y}$ avait eu opérations frauduleuses (R. M. Breckenridge, « The Canadian Banking System », Journal of the Canadian Bankers' Association, Vol. II, No 11, (Déc. 1894) : 166). La « Farmers Joint Stock Banking Company », établie en 1835 pour faire concurrence à l'aristocratique «Bank of Upper Canada 》, était appuyée par l'élément libéral que dirigeait William Lyon Mackenzie, le premier maire de Toronto. La Banque pratiquait encore ses affaires en 1849, mais il semble qu'elle ait cessé d'exister vers cette époque. Deux des personnes qui ont fondé cette Banque s'en sont séparées et, en 1835, ont fondé une banque semblable, intitulée \& The Bank of the People», qui a vécu jusqu'en 1838 (Breckenridge, loc. cit., p. 152). La quatrième institution, la Banque du Peuple, établie en 1835, n'était guère une banque du peuple; elle fut fondée par l'institution bancaire française de Viger, DeWitt et Cie, et elle connut des jours prospères (Ibid: 133-134). (Voir également: Adam Shortt, "The History of Canadian Currency, Banking and Exchange», Journal of the Canadian Bankers' Association, Vol. VIII, No 4, (Juillet 1901) : 315-326). Shortt mentionne également deux projets ingénieux de banques hypothécaires en 1835 et 1837; mais il est douteux qu'elles aient jamais exercé d'opérations (Shortt, loc. cit., vol. IX, No 1, (Oct. 1901) : 5-7). Toutefois, il appert, d'après la description qu'en donne Shortt, que ces banques n'étaient que des institutions de spéculation, et non pas des banques du peuple telles que définies au présent article. 
ont été expulsés de leurs foyers et de leurs fermes. ${ }^{3}$ Quelques-uns ont trouvé dans les bois, refuge contre les soldats britanniques qui les pourchassaient, ou sont revenus d'exil. Ce groupe qui, en 1768 , ne comptait que 203 personnes, y compris 123 enfants, a constitué le noyau de la communauté acadienne de l'Île du Prince-Édouard. Ces gens vivaient dans un état voisin de la famine, car leurs demeures et leurs troupeaux avaient été complètement anéantis. Ceux qui avaient échappé à la déportation étaient considérés comme des prisonniers et en réalité, esclaves de leurs conquérants. Durant nombre d'années, tous droits politiques et civils leur furent refusés. On les exploitait honteusement; parfois on leur permettait de défricher et de cultiver des terres, mais ils pouvaient en être expulsés par des colons anglais qui s'étaient procuré des titres légaux. Les Acadiens ont dû abandonner les meilleures terres et s'établir dans des régions moins fertiles. Cette situation a duré pendant plusieurs générations. ${ }^{4}$

Toutefois, la communauté acadienne a démontré qu'elle pouvait survivre et prospérer malgré l'oppression et l'exploitation. Ces Acadiens ont puisé leur force dans leurs croyances religieuses; autour de la paroisse, ils ont organisé une vie collective. Leur langue, également, était un autre lien. Petit à petit, leur sort s'est amélioré, et un siècle après la déportation, ils étaient groupés en sept paroisses, dont Rustico est la plus ancienne. ${ }^{5}$ L'exiguïté de l'île les a empêchés de se disperser, et ils ont établi des relations commerciales et politiques avec leurs voisins d'origine anglaise, écossaise et irlandaise. Vers le milieu du dixneuvième siècle, ils s'étaient assez bien intégrés à la vie économique de la province. Parce que le problème de la propriété des terres n'avait pas été réglé, tous les cultivateurs de l'Île du Prince-Édouard vivaient dans un état d'incertitude. Cependant, la paroisse constituait le centre de la vie sociale des Acadiens

3 A. B. Warburton, A History of Prince Edward Island (St. John, N.B.: Barnes and Co., Imprimeurs, 1923), 84-94.

4 J.-H. Blanchard, Histoire des Acadiens de l'Ile du Prince-Edouard (Moncton, N.-B.: Imprimerie de l'Evangile, 1927), 34-38.

5 Ibid, 55. 
qui, au milieu du dix-neuvième siècle, étaient pauvres, illettrés, humbles, mais dévoués.

A cette époque, en 1859, en la paroisse de Rustico, vint un pasteur, l'abbé Georges-Antoine Belcourt, qui sut guider vigoureusement cette population non seulement dans ses croyances religieuses, mais également dans le domaine économique. Né à Québec où il avait fait ses études, il était déjà l'un des grands missionnaires de l'Ouest américain. Il avait compilé l'une des premières grammaires de la langue que parlaient les tribus indiennes vivant dans la région de la Rivière-Rouge. Ses rapports avaient été présentés au Congrès des États-Unis. Quelques-uns de ses exploits étaient presque légendaires, tel le voyage qu'il effectua au milieu de l'hiver, de Minnesota à Saint-Louis, afin d'obtenir des médicaments, alors qu'une épidémie sévissait chez les Indiens. ${ }^{6}$ Rappelé de l'Ouest, l'abbé Belcourt fut nommé curé de la paroisse de Rustico, où il initia un mouvement de renaissance culturelle et économique. De Québec, il fit venir un éducateur, et fonda une école supérieure destinée aux jeunes de talent de la région; il organisa une fanfare et une chorale; pour les adultes, il organisa un «institut » où l'on étudiait les méthodes techniques agricoles et dont on ne pouvait être membre si l'on n'était abstème; il fonda une bibliothèque, et l'Empereur Napoléon III lui accorda un octroi annuel de 1,000 francs pour l'achat de livres. ${ }^{7}$

Fondée alors que l'abbé Belcourt était curé, la « Farmers' Bank of Rustico » constituait, dans le domaine économique, une des phases de la résurrection générale d'un peuple. Il faut admirer l'audace d'un homme qui, dans les conditions qui prévalaient alors, avait songé à fonder une banque à charte. A cette époque, dans l'Empire Britannique, la Banque était un domaine réservé à l'élite des classes commerciales. Comme nous le constaterons

${ }^{6}$ Allen Johnson, Ed., Dictionary of American Biography (London, 1929), II : 145; R. P. LeJeune, Dictionnaire Général ... du Canada (Ottawa: 1931), I: 152-153. James M. Reardon, George Anthony Belcourt, Pioneer Catholic Missionary of the Northwest, 1803-1874 (St. Paul, Minn.: North Central Publishing Company, 1955), 171-185.

7 J.-H. Blanchard, Rustico: une paroisse acadienne de l'Ile du PrinceEdouard (Volume souvenir, Rustico: 1938), 47-48. 
dans un extrait de ses lettres, l'abbé Belcourt était au courant des développements apportés aux banques du peuple en France, où, dès 1850 et même 1840 , on avait tenté de fonder des banques du peuple. L'Empereur lui-même avait encouragé ce mouvement. La législation fondamentale concernant le crédit sur les terres, en France, date d'une loi de $1852 .{ }^{8}$

Mais l'abbé Belcourt était plus qu'un érudit, plus qu'un organisateur ou un promoteur: il avait une connaissance approfondie de la nature humaine. Ses longs séjours au milieu des tribus indiennes lui avaient sans doute révélé l'importance qu'exercent les symboles dans la vie des hommes. Même avant que la population de la paroisse n'ait demandé une banque à charte, il avait engagé ses paroissiens à construire un local convenable où loger cette banque future. Ses ouailles avaient la foi; sur le sommet de la colline, ils avaient une église en bois. Mais il fallait que leur banque, plus bas sur le flanc, soit construite en pierre de taille. Les maisons en pierre sont très rares dans l'île du PrinceÉdouard. Terminé en 1861, cet édifice est encore aussi solide qu'au jour où il a été érigé. C'est un immeuble de deux étages, mesurant environ 33 pieds par 50 pieds; les murs sont en pierre rouge d'une épaisseur de 14 pouces; les poutres de 12 pouces ont été taillées à la hache, et le reste de l'édifice est aussi solide; la charpente du toit a été posée sans clous, et de nombreuses fenêtres assurent un éclairage convenable. Cet édifice est un symbole de force et de solidité. Le bureau de la banque, ainsi que la bibliothèque, étaient au rez-de-chaussée; au deuxième, se trouvait une salle de réunions publiques. ${ }^{9}$ Outre sa signification symbolique, cet édifice a grandement contribué au succès des opérations de la Banque; parce qu'elle ne payait pas loyer pour ses locaux, la Banque n'avait aucune charge fixe à assumer et pouvait ainsi consacrer tous ses revenus à des prêts ou les placer comme actif liquide.

8 Myron T. Herrick et R. Ingalls, Rural Credits: Land and Cooperative (New York and London: D. Appleton and Company, 1916), 111-127, 321-327.

$9 \mathrm{Au}$ cours de l'été de 1954, l'auteur, accompagné par le docteur J.-H. Blanchard, l'historien acadien, examina tout l'immeuble, du grenier jusqu'à la cave. 
Avant l'époque de l'abbé Belcourt, et en réalité depuis la fin $\mathrm{du}$ dix-huitième siècle, l'île du Prince-Édouard avait souffert d'une insuffisance chronique de monnaie. A ce sujet, Ross exprime l'opinion suivante:

Il semble que l'Ile du Prince-Edouard ait constitué un champ fertile où tenter des expériences monétaires, sans doute, dans une certaine mesure, parce qu'une population si peu nombreuse et qui s'adonnait presque exclusivement à l'agriculture et à la pêche, pouvait difficilement trouver un genre de devises qui puissent demeurer à l'intérieur de l'Ile. ${ }^{10}$

Apparemment, l'économie de l'île reposait dans une grande mesure sur l'emploi du crédit. Les journaux de l'époque 1860 mentionnent souvent le crédit accordé par les magasins, la perception de comptes et autres sujets de ce genre. L'omniprésente compagnie «Singer Sewing Machine » offrait ses produits «au comptant ou contre un bon billet ».11 En 1860, il n'existait qu'une banque dans l'île du Prince-Édouard, qui avait obtenu sa charte en $1856 .{ }^{12}$ On a allégué que cette banque exigeait un taux d'intérêt de 71/2 pour cent.13 Mais la population et les affaires augmentant, la Banque de l'île du Prince-Édouard ne pouvait prêter à tous ceux qui désiraient s'adresser à elle. ${ }^{14}$ L'escompte à taux usuraire et l'usure étaient de pratique courante. En 1863, dans un éditorial du journal «Islander », on pouvait lire ce qui suit: «... celui qui escompte à titre privé peut facilement placer tous ses deniers dans des valeurs sûres et à son propre taux d'intérêt ».15 L'année suivante, lors d'un débat sur une loi visant à contrôler le taux d'intérêt exigé dans l'île - en réalité cette loi avait pour but d'abroger la loi d'usure qui limitait le taux d'intérêt à six pour cent - tous les orateurs conviennent que la loi régissant l'usure n'est nullement appliquée. D'après ce que rap-

10 Victor Ross, A History of the Canadian Bank of Commerce (Toronto: Oxford University Press, 1920), I: 127-128; 423-430.

i1 The Islander, Charlottetown, Ile du Prince-Edouard, 12 août 1864.

12 Howard, op. cit., 30.

13 The Islander, 22 avril 1864.

14 Ibid., 17 avril 1863.

15 Ibid., 12 juin 1863. 
porte le journal «Islander », un orateur a dit: «Le taux légal est présentement de six pour cent; mais on contourne la loi en acceptant des billets à ordre à un taux d'intérêt supplémentaire de 20 à 25 pour cent ». D'autres ont mentionné des taux d'intérêt de 15 à 20 pour cent. ${ }^{16} \mathrm{Il}$ était évident qu'il fallait accroître les facilités bancaires dans l'île du Prince-Édouard.

Il semblait que les conditions politiques et économiques se prêtaient alors à la constitution en corporation d'une nouvelle banque. La monnaie était rare et le crédit cher; la colonie venait d'obtenir un gouvernement responsable, et la question de la propriété des terres inquiétait la population. ${ }^{17}$ Le lundi 30 mars 1863, on lisait dans le journal «Islander »: «L'Hon. M. Walker a présenté une pétition de certains habitants de Rustico et de la région avoisinante, demandant l'adoption d'une loi qui constitue en corporation une banque qui sera dénommée The Bank of Farmers of Prince Edward Island $\gg .^{18}$

La loi constituant en corporation la «Farmers' Bank of Rustico » a suivi le cours régulier de toute mesure législative, et la législature l'a finalement adoptée le 21 avril $1863 .{ }^{19}$ Toute-

16 Ibid., $1^{\mathrm{er}}$ avril 1864.

17 Carl Wittke, A History of Canada (New-York: F. S. Crofts and Co., 1942), 131-132.

18 The Islander, 10 avril 1863.

19 Journal de la Chambre d'Assemblée de l'Ile du Prince-Edouard (Charlottetown, 1863) :

a) Pétition de Fabien Doucet et d'autres personnes, demandant l'adoption d'une loi intitulée: «An Act to incorporate a Joint Stock Banking Company at Rustico 》 (p. 90) ;

b) Loi visant à constituer en corporation diverses personnes sous le nom de Président, Administrateurs et Compagnie de la Farmers' Bank of Rustico - rapport du Comité et première lecture (p. 107);

c) Bill renvoyé au Comité des bills privés (p. 107);

d) Présentation du rapport (pp. 112-113);

e) Prise en considération du rapport (pp. 121, 139) ;

f) Le rapport n'est pas adopté, et le bill est de nouveau renvoyé en comité (p. 168);

g) Le bill est lu une troisième fois, modifié et adopté (p. 170) ;

h) Adoption par le Conseil (p. 185);

i) Sanction (p. 192).

Journal du Conseil Législatif de l'Ile du Prince-Edouard (Charlottetown, 1863) :

a) Loi constituant en corporation la Farmers' Bank, présentée et lue la première fois (p. 109); 
fois, la loi n'a été confirmée qu'une année plus tard. Au mois de novembre de cette année, Lord Newcastle, le secrétaire d'État aux colonies, a demandé au lieutenant-gouverneur de lui fournir des renseignements supplémentaires concernant le capital de cette banque. Il a été renversé d'apprendre que le capital initial avait été établi à la minime somme de $£ 1,200$. En monnaie de l'Île, $£ 1,200$ valaient 800 livres Sterling, soit moins de $\$ 3,900$. Mais une autre disposition du bill autorisait la Banque à augmenter son capital jusqu'à $£ 21,200$. «Les Lords ont signalé que ces dispositions extraordinaires ne garantissent nullement la responsabilité des promoteurs de ce projet; et avant que cette loi soit soumise à l'approbation de Sa Majesté, ils désirent obtenir un rapport sur cette question...» (Voilà ce qu'écrivait Lord Newcastle au lieutenant-gouverneur.) ${ }^{20}$

Après un certain retard, le Gouverneur transmit à Lord Newcastle, le 30 janvier 1864, un rapport que lui avait adressé le procureur général de l'île, lequel rapport ne mentionnait que les représentations de la population de Rustico:

... Ils savent respectueusement que la garantie couvrant la responsabilité des promoteurs du projet ne doit pas reposer sur la modicité du capital initial projeté ni sur le montant du permis qui l'augmentera, mais, comme dans les autres lois concernant les banques, elle est sujette aux dispositions de la loi même...

Les Lords étaient d'avis que les dispositions de la loi couvrant la responsabilité des actionnaires s'appliquaient de façon aussi sévère à l'égard de cette banque qu'à l'égard des deux

b) Deuxième lecture, renvoi en comité et rapport de l'état de la question (p. 116) ;

c) De nouveau le bill est renvoyé en comité, et rapport de l'état de la question (p. 117) ;

d) De nouveau, renvoi en comité et rapport (p. 118) ;

e) Troisième lecture, adoption et message à l'Assemblée (p.122) ;

f) Sanction par le Lieutenant-Gouverneur (p.131).

Débats et Procès-verbaux de l'honorable Conseil Législatif de l'Ile du Prince-Edouard - Session de 1863 (Charlottetown, 1863), 33, 53, 59-61.

20 Correspondance entre le Duc de Newcastle, secrétaire d'Etat, et le Lieutenant-Gouverneur Dundas (Journaux du Conseil Législatif de l'Ile du Prince-Edouard, 27 Victoria, $2^{\mathrm{e}}$ Session de la $22^{\mathrm{e}}$ Assemblée Générale (Charlottetown, 1864), Appendice no 2, 24-27). 
autres banques que la législature de l'Île avait constituées en corporation. Ils ont même signalé que deux clauses du bill concernant la Banque de Rustico étaient plus rigoureuses : cette dernière ne pouvait pratiquer ses opérations avant que la totalité du capital n'ait été versée en or ou en argent, alors que les deux autres banques ne devaient verser qu'un tiers de leur capital avant de commencer leurs opérations. En outre, cette banque ne pouvait émettre des billets pour une valeur supérieure au double du montant de son capital social, alors que les deux autres banques pouvaient le faire jusqu'à concurrence de trois fois leur capital social. ${ }^{21}$

Ces motifs ont prévalu, et le lieutenant-gouverneur recevait un document daté du 13 avril 1864 l'informant que Sa Majesté avait sanctionné ce bill le 7 avril 1864 :

... Ce jour, sur l'avis du Conseil Privé, il a plu

à Sa Majesté de déclarer qu'elle accordait confirmation spéciale à ladite loi, et ladite loi est par la présente spécialement confirmée, ratifiée et en conséquence finalement mise en vigueur. ${ }^{22}$

C'est la plus petite banque qui ait jamais obtenu une charte dans le territoire qui subséquemment devait faire partie de la Confédération, ${ }^{23}$ et probablement la plus petite banque à charte de tout l'Empire Britannique.

La loi en vertu de laquelle la «Farmers' Bank of Rustico» a été constituée en corporation était une mesure législative de portée générale. ${ }^{24}$ Le premier article de la loi accordait à la com-

21 Ibid.

22 Journal de la Chambre d'Assemblée de l'Ile du Prince-Edouard (Charlottetown, 1864), 140 et Appendice X. Confirmation en a été publiée dans la Gazette Royale officielle, le 4 mai 1864.

23 B. H. Beckhart, The Banking System of Canada, reproduit de Foreign Banking System (New-York, Henry Holt and Company, 1929), 334-337. Ces pages renferment un tableau indiquant le capital des banques canadiennes qui, entre 1817 et 1926, ont cessé leurs opérations. (En 1926, il ne restait que dix grandes banques.) La « Farmers' Bank of Rustico 》 possédait sans contredit le capital le plus minime. Incidemment, la deuxième plus petite banque était la Banque de Summerside, également de l'Ile du Prince-Edouard; mais elle était six fois plus considérable que la Banque de Rustico.

${ }_{24}$ Lois privées et locales de l'Assemblée Générale de l'Ile du PrinceEdouard, 26 Victoria, 1863, Chap. XVI: 28-41. 
pagnie les pouvoirs généraux d'une corporation; l'article 3 énonçait en détail les pouvoirs de cette corporation: permission de détenir des terres et terrains dont la valeur ne dépasse pas $£ 1,500$, de prendre des biens immeubles en recouvrement d'une dette, et d'accepter des hypothèques et autres garanties. L'article 18 autorisait la corporation à effectuer toutes opérations concernant les lettres de change, les billets à ordre, l'or et l'argent en lingots, ainsi que la vente d'objets nantis. L'article 2 établissait le capital social à $£ 1,200$, divisé en actions de $£ 1$ chacune, et « devant être payées en pièces d'or ou d'argent ayant cours dans l'Île, durant l'année qui suivra l'adoption de la présente loi ... » L'article 13 stipulait que les souscriptions seraient restreintes à cinquante actions durant les premiers six mois de l'entrée en vigueur de la loi, mais que le nombre en pourrait être augmenté à cent après six mois, si alors la totalité des actions n'avait pas été souscrite. Un actionnaire ne pouvait détenir plus de 300 actions, ou plus de 10 pour cent du capital social. L'article 15 interdisait à la Banque de commencer ses opérations avant que la totalité des $£ 1,200$ ait été versée. L'article 16 stipulait qu'une fois cette somme versée, le Secrétaire aux colonies nommerait trois commissaires chargés de compter l'argent et de recevoir des administrateurs une déclaration sous serment à l'effet que cet argent constituait le capital de la Banque. Pour toutes fins pratiques, c'était là le seul contrôle que le gouvernement exerçait sur la Banque; toutefois, l'article 29 autorisait un comité de la législature à examiner les livres de la Banque, et l'article 33 stipulait quels renseignements le caissier devait soumettre au Secrétaire aux colonies à tous les six mois. Ces renseignements étaient publiés sous forme d'appendices dans le Journal de l'Assemblée Législative. Ainsi, un rapport imprimé rendait publiques, en quelque sorte, les opérations de la Banque. Aucune vérification extérieure n'était requise, mais l'article 23 stipulait que les administrateurs pouvaient examiner les livres; et ce privilège était refusé aux actionnaires ordinaires.

Les articles 4 à 9 , ainsi que les articles 14,32 et 34 sont d'ordre technique et concernent les qualités et les pouvoirs des administrateurs. Il devait y avoir 12 administrateurs, dont six 
élus chaque année, et ces administrateurs devaient élire un président parmi eux. Huit administrateurs constituaient quorum et on pouvait leur accorder rémunération, avec l'approbation des actionnaires. Un actionnaire pouvait devenir administrateur à condition qu'il ne soit pas administrateur d'une autre banque. Les administrateurs étaient autorisés à remplir les vacances survenant parmi les membres du conseil d'administration et à engager des commis. En vertu de l'article 10, le caissier et les commis devaient fournir un cautionnement «proportionné à la confiance qui leur était dévolue ». Un administrateur qui a manqué d'acquitter un prêt ne pouvait siéger à une assemblée du conseil d'administration. Le montant global des dettes des administrateurs ne devait pas dépasser, en aucun temps, dix pour cent du capital social.

L'article 11 établissait un droit de vote d'après une échelle graduée: jusqu'à neuf actions, un vote; pour chaque dix actions, deux votes; pour chaque vingt actions dépassant dix, un vote supplémentaire, jusqu'à concurrence d'un maximum de quinze votes. L'article 12 admettait des fondés de pouvoirs, mais sous réserves. D'après l'article 17 , les actions pouvaient être cédées. En vertu de l'article 19, les actionnaires « étaient tenus de payer une somme dépassant deux fois le montant de capital qu'ils détenaient alors ... au delà et outre le montant de capital qu'ils avaient effectivement versé à la banque »; l'article 20 stipulait que la même disposition s'appliquait à l'égard des billets de banque. L'article 21 limitait à deux fois le montant du capital social la somme totale des dettes de la Banque, soit en billets, soit en lettres de change.

Les billets devaient être signés par le président et le caissier et imprimés sur plaques d'acier (article 24); et la Banque s'engageait à honorer les billets contrefaits (article 25). L'article 38 interdisait à la Banque de consentir un prêt sur la garantie de son propre capital.

L'article 26 stipulait que la Banque devait être située à Rustico; l'assemblée annuelle devait être tenue le premier jeudi de juillet, et l'article 27 spécifiait ce qui devait être soumis à 
l'assemblée. D'après l'article 22, les administrateurs devaient verser des dividendes trimestriels.

La plupart des autres dispositions de la loi sont d'ordre technique: assemblées extraordinaires (article 30) ; procédures pour dissolution (article 31); suspension des paiements en espèces (article 43); sanction royale (article 45 ); poursuites concernant billets à ordre (article 35); actions de banque comme biens personnels (article 36); saisie d'actions au même titre qu'autres biens personnels (article 37) ; et dispositions d'ordre technique pour accroître le capital (article 38 à 42). La dernière disposition prévoyait une méthode d'augmenter, avec l'approbation des actionnaires, le capital social, jusqu'à concurrence de $£ 21,200$, divisé en actions d'une valeur au pair d'une livre chacune. L'article 44 accordait à la Banque une charte de 20 années, qui devait expirer le 1er juin 1883.

Voilà, en résumé, la loi constituant en corporation la «Farmers' Bank of Rustico». Sauf la valeur minime de ses actions au pair ainsi que de son capital, la plupart des dispositions de la loi étaient identiques à celles qui s'appliquaient aux banques commerciales à charte exerçant alors leurs opérations dans l'Ile du Prince-Edouard. Cette banque avait obtenu une charte de banque commerciale, mais aucune disposition de sa charte ne lui interdisait de pratiquer les opérations d'une banque du peuple.

M. Jérôme Doiron, cultivateur, fut le premier président de la Banque, et M. Marin Blanchard, instituteur, en était le caissier. La Banque était ouverte une après-midi par semaine, alors que les administrateurs se réunissaient aux fins d'étudier les demandes de prêts. Et ainsi, il a été possible de réduire le taux d'intérêt aux emprunteurs. ${ }^{25}$ Les billets de cette banque

25 Victor Ross: A History of the Canadian Bank of Commerce, I: 151. Ross affirme que la « Farmers' Bank of Rustico 》 a réduit le taux d'intérêt jusqu'à 7 ou 8 pour cent. Bien que ce chiffre soit probablement exact, le bref exposé de Ross relatif à la Banque de Rustico contient tellement d'inexactitudes qu'il faut accepter avec prudence le taux d'intérêt qu'il mentionne. 
étaient, en général, acceptés partout; ainsi, le gouvernement acceptait n'importe quel montant de billets provenant de la Banque de Rustico, alors qu'elle n'acceptait les billets de la «Summerside Bank » qu'à concurrence de $£ 100$. Un agent de la Banque de Rustico venait à Charlottetown une ou deux fois par semaine afin de racheter et échanger les billets de banque. ${ }^{26}$ Voilà la situation qui existait vers la fin des années 1860, alors que l'abbé Georges Belcourt était curé de Rustico.

L'abbé Belcourt a quitté Rustico en 1869 afin d'occuper un nouveau poste aux Îles-de-la-Madeleine. Au début des années 1870 , il était rumeur que la situation financière de la Banque était compromise. Evidemment, les journaux de l'Ile n'ont pas mentionné ce sujet; durant ces années, les journaux locaux n'ont que rarement fait allusion à la Banque de Rustico - et en réalité à quelque banque que ce soit. On peut vérifier le bienfondé de ces rumeurs concernant la Banque en lisant les extraits suivants d'une lettre que l'abbé Belcourt a écrite, en novembre 1873, au journal Le Moniteur Acadien, publié à Shédiac, Nouveau-Brunswick, et qui était grandement répandu parmi la population acadienne. ${ }^{27}$

En 1873, l'abbé Belcourt quitta les Îles-de-la-Madeleine pour Memramcook, Nouveau-Brunswick. Lorsqu'il eut vent des rumeurs défavorables concernant la Banque de Rustico, il avisa le caissier de préparer ses livres pour fins de vérification. Subséquemment, dès qu'il en eut l'occasion, l'abbé Belcourt se rendit à Rustico. A son retour, il écrivit au rédacteur du journal $L e$ Moniteur Acadien une lettre dans laquelle, après avoir expliqué pourquoi il était allé à Rustico, il déclarait spécifiquement ce qui suit:

Je désire aujourd'hui rassurer l'opinion publique sur l'état de cette institution, que je regarde comme d'une importance vitale pour le Fermier. J'ai donc la satisfaction de pouvoir dire que tous les livres, comptes, arrérages, dettes douteuses, etc., examinés, cette Banque est parfaitement sure et

26 The Herald, Charlottetown, Ile du Prince-Edouard, 16 mai 1866.

27 Le Moniteur Acadien, Shédiac, Nouveau-Brunswick, 13 novembre 1873. 
peut rencontrer et faire honneur à ses obligations, tellement que s'il plaisait à ses directeurs de cesser de fonctionner, tous les actionnaires recevraient leurs capitaux complets. Il y a des pertes probables, mais ces pertes ne peuvent affecter que la caisse de réserve, résultant de fractions de dividendes qui sont comparativement considérables vu que tout le capital émissible est toujours en circulation, ce capital étant au dessous de la demande, ne peut rester oisif. ${ }^{28}$

Et subséquemment, dans sa lettre, l'abbé Belcourt expose ses vues concernant les banques du peuple - ou les banques foncières, comme il les appelait:

Qu'il me soit permis de faire remarquer au public en général et aux institutions financières du commerce, qu'une Banque foncière étant d'une importance vitale à la prospérité de l'agriculture, mérite toute la faveur possible de la part des banques commerciales, ses consœurs. C'est le fermier qui crée la richesse, c'est le père nourricier de la société tout entière, c'est de lui que le boulanger reçoit sa farine, le tisserand sa laine, le cordonnier son cuir, le boucher son bœuf, etc. Le fermier est de tous les membres de la société celui qui porte le fardeau le plus lourd; il a donc besoin d'une nourriture forte et mérite l'affection, l'assistance et la sympathie de tout homme bien pensant. Oter au fermier sa banque ce serait le faire tomber dans les griffes avides de l'usurier (Broker), cette sangsue publique qui tient à l'état de misère cette classe si intéressante des fermiers. Il n'y a qu'une banque qui puisse prêter les plus petites sommes comme les plus fortes à des taux modérés, et leur venir en aide pour l'acquisition d'un bien-fonds. Favorisons l'essor de ces jeunes bras vigoureux qui ne demandent qu'un fond pour réaliser cette richesse latente que leur vivace énergie fera sortir du sol.

Et l'abbé Belcourt continue à expliquer la technique des opérations des banques du peuple:

Si l'on remarque combien il est difficile à une Banque foncière de subsister longtemps, si on l'as- 
sujettit à une rigueur impitoyable, je dirais: seraitil donc impossible de faire entre les banques quelques conventions en faveur des Banques foncières ? Dans les classes commerciales, l'argent roule sur les comptoirs tous les jours et en tout temps; peu leur importe qu'il leur faille payer leur emprunt tous les trois mois; mais chez le fermier, le revenu de ses capitaux prend la lenteur de la végétation; ce n'est qu'après sa récolte que le fermier peut remplir de forts paiements; il faut donc de toute nécessité une manœuvre différente et fort pénible à ses officiers pour guider et conduire à bonne fin les opérations de ces sortes d'institutions, dont on a senti partout l'importance; que l'on a essayé d'établir partout en Europe et ici; et qui ont été abandonnées à cause du manque de constance (en toute probabilité) puis à cause de l'assiduité requise et des dégoûts à rencontrer de la part des banques commerciales. ${ }^{29}$

L'abbé Belcourt terminait sa lettre en assurant au public que les banques du peuple sont établies selon des normes de toute sécurité, et il préconisait fortement l'organisation de banques du peuple supplémentaires dans d'autres localités « où les mêmes besoins se font sentir ».

L'abbé Belcourt a compris le problème, et il en a trouvé la solution: un grand nombre de banques du peuple exerçant leurs opérations partout dans la Nouvelle Acadie. Une de ces banques du peuple, la «Farmers' Bank of Rustico », a eu la vie dure; mais un mouvement de ce genre, tel qu'il existait alors en Europe, ne pouvait que réussir. Mais l'abbé Belcourt, après les nombreuses années dures qu'il avait passées dans les pays missionnaires, de la Baie d'Hudson à la Rivière-Rouge, était alors âgé et fatigué, et en outre, il avait déjà vécu dix années parmi les Acadiens de l'est du Canada. Il avait prédit un avenir brillant aux banques du peuple; mais il a fallu l'arrivée d'Alphonse Desjardins, une génération plus tard, pour ranimer dans la province de Québec ce mouvement alors connu sous le nom de Caisses Populaires, qui était en voie de périr dans les provinces maritimes.

29 Ibid. 
En effet, au moment même où l'abbé Belcourt écrivait cette lettre, la Banque de Rustico était condamnée à disparaître. L'Île du Prince-Édouard était entrée dans la Confédération au mois de juillet précédent, et ainsi le gouvernement provincial avait cédé le contrôle qu'il exerçait sur les banques et les opérations bancaires; car l'Acte de l'Amérique du Nord britannique stipule que le gouvernement fédéral a pouvoir exclusif dans ce domaine. ${ }^{30}$ En vertu de la loi de 1871 concernant les banques, le gouvernement fédéral avait décidé d'abolir les petites banques sans succursales et d'instituer un régime de banques avec succursales dans tout le pays. Cette loi établissait à $\$ 500,000$ le capital minimum d'une banque à charte, dont au moins $\$ 100,000$ devaient être versés avant que la banque ne commence ses opérations, et un autre $\$ 100,000$ dans les deux années suivantes. ${ }^{31}$ Les petites banques ne pouvaient faire autrement que vendre leur actif à leurs puissants concurrents. Cependant, le gouvernement fédéral a constaté qu'il ne pouvait pas facilement liquider la Banque de Rustico: durant vingt années, elle a survécu malgré le gouvernement fédéral et les pouvoirs accordés aux grandes banques commerciales.

\section{III}

Mais avant de retracer l'histoire des mesures législatives concernant cette banque, examinons-en les opérations financières. $^{32}$ L'abbé Belcourt n'exagérait pas lorsqu'il déclarait que

30 Acte de l'Amérique du Nord britannique, 1867, 30 et 31 Victoria, Chap. III, Art. 91, No 15 .

31 Beckhart, op cit., 298-302.

32 Les états financiers qui ont pu être reconstruits l'ont été d'après les rapports que l'article 33 de la Loi exigeait de soumettre au Secrétaire aux Colonies. Ces états étaient déclarés sous serment devant un juge de paix. Apparemment, la Banque négligeait de déposer ces rapports, car les Appendices du Journal de la Chambre d'Assemblée indiquent qu'elle n'a fait rapport que pour les années $1866,1867,1871,1873,1874$ et 1875 . Durant cette période de dix années, les autres banques de l'Ile produisaient régulièrement leurs rapports annuels. Après qu'une loi fédérale eut prorogé, en 1883, la charte de la Banque de Rustico, il a été ordonné à cette dernière de continuer à soumettre ses rapports au ministre des Finances. Ils apparaissent dans la Gazette du Canada de 1885 à 1891. Cependant, les statistiques concernant la Banque de Rustico ne sont pas incluses dans les études détaillées publiées sur les banques à charte: e.g. Report of the Chartered 


\section{TABLEAU No 1}

«Farmers' Bank of Rustico», Ile du Prince-Edouard, Bilans comparatifs pour certaines années 1866-1891 1

\begin{tabular}{|c|c|c|c|}
\hline . & $\begin{array}{c}2 \\
1866 \\
3 \text { avril }\end{array}$ & $\begin{array}{c}3 \\
1867 \\
4 \text { juil. }\end{array}$ & $\begin{array}{c}4 \\
1871 \\
4 \text { avril }\end{array}$ \\
\hline $\begin{array}{l}\text { PASSIF } \\
\text { Capital social } \\
\text { Billets en circulation } \\
\text { Sommes dues à la « Union Bank } \\
\text { of P.E.I. » } \\
\text { Sommes dues sur dividendes } \\
\text { Dépôts portant intérêts }\end{array}$ & $\begin{array}{c}\$ 4,549 \\
14,132 \\
- \\
\overline{1,809}\end{array}$ & $\begin{array}{r}\$ 8,202 \\
15,682 \\
284 \\
-659\end{array}$ & $\begin{array}{r}\$ 8,870 \\
15,652 \\
746 \\
\overline{2,135}\end{array}$ \\
\hline Totalité du passif & $\$ 20,490$ & $\$ 24,827$ & $\$ 27,403$ \\
\hline $\begin{array}{l}\text { ACTIF } \\
\text { Or et argent } \\
\text { Billets du Dominion et autres } \\
\text { billets de banque } \\
\text { Sommes dues à la Banque }\end{array}$ & $\begin{array}{r}\$ 8,399 \\
283 \\
12,068\end{array}$ & $\begin{array}{r}\$ 3,439 \\
472 \\
21,404\end{array}$ & $\begin{array}{r}\$ 3,823 \\
189 \\
23,555\end{array}$ \\
\hline Totalité de l'actif & $\begin{array}{r}\$ 20,750 \\
20,490\end{array}$ & $\begin{array}{r}\$ 25,315 \\
24,827\end{array}$ & $\begin{array}{r}\$ 27,567 \\
27,403\end{array}$ \\
\hline Montant à l'actif de la Banque & $\$ 260$ & $\$ \quad 488$ & $\$ \quad 164$ \\
\hline
\end{tabular}

PASSIF

Capital social

Billets en circulation

Sommes dues à la « Union Bank of P.E.I. 》

Sommes dues sur dividendes Dépôts portant intérêts

Pourcentages de la totalité du passif et de l'actif

\begin{tabular}{lrrr} 
& 100.0 & 100.0 & 100.0 \\
\cline { 2 - 4 } & & & \\
\hline $\begin{array}{c}\text { ACTIF } \\
\text { Or et argent }\end{array}$ & 40.5 & 13.6 & 13.9 \\
$\begin{array}{l}\text { Billets du Dominion et autres } \\
\text { billets de banque }\end{array}$ & 1.4 & 1.9 & .7 \\
Sommes dues à la Banque & 58.1 & 84.5 & 85.4 \\
\cline { 2 - 4 } & 100.0 & 100.0 & 100.0 \\
$\begin{array}{c}\text { Montant à l'actif de la Banque } \\
\text { en pourcentage de l'actif }\end{array}$ & 1.25 & 1.92 & .59 \\
\hline
\end{tabular}

1 Les bilans ont été disposés de façon à correspondre à la méthode employée dans la Gazette du Canada durant les années 1885 à 1891. Les totaux ont été établis au chiffre le plus près. Les chiffres couvrant les années 1866, 1867 et 1871 ont été transposés en dollars au taux de $\$ 3.2444$ par livre. C'est à peu près le même taux de $\$ 3.244 / 9$ que prescrit la Loi concernant les banques et le commerce de banque, 34 Vict., c. 5, art. 6. Etant donné que cent livres sterling valaient cent cinquante livres en monnaie de l'Ile, les chiffres ci-haut mentionnés correspondent à la valeur-or du dollar américain. En ce qui concerne les taux de change de monnaies en cours dans les provinces maritimes, ainsi que la Loi concernant les banques et le commerce de banque, voir Lieut.-Gouverneur Robinson, Rapport annexé au Livre bleu de l'Ile du Prince-Edouard pour l'année 1870 (Charlottetown - Bremmer Brothers, Imprimeurs, 1871), pp. 51 à 53. 
TABLEAU No 1 (suite)

«Farmers' Bank of Rustico», Ile du Prince-Edouard, Bilans comparatifs pour certaines années 1866-1891 1

\begin{tabular}{|c|c|c|c|c|c|}
\hline 5 & $\begin{array}{c}6 \\
1875\end{array}$ & $\begin{array}{c}7 \\
1885\end{array}$ & $\begin{array}{c}8 \\
1887\end{array}$ & $\begin{array}{c}9 \\
1890\end{array}$ & $\begin{array}{c}10 \\
1891\end{array}$ \\
\hline $\begin{array}{c}1873 \\
1^{\text {er }} \text { avril }\end{array}$ & $\begin{array}{c}1875 \\
6 \text { avril }\end{array}$ & $\begin{array}{l}1885 \\
6 \text { oct. }\end{array}$ & $\begin{array}{l}1887 \\
4 \text { oct. }\end{array}$ & $\begin{array}{l}1890 \\
7 \text { oct. }\end{array}$ & $\begin{array}{l}1891 \\
6 \text { oct. }\end{array}$ \\
\hline $\begin{array}{r}\$ 8,870 \\
13,625\end{array}$ & $\begin{array}{r}\$ 8,834 \\
11,760\end{array}$ & $\begin{array}{r}\$ 8,212 \\
12,217\end{array}$ & $\begin{array}{r}\$ 8,212 \\
11,755\end{array}$ & $\begin{array}{r}\$ 8,212 \\
10,466\end{array}$ & $\begin{array}{r}\$ 8,212 \\
12,488\end{array}$ \\
\hline 126 & 1,032 & - & - & - & - \\
\hline$\overline{2,018}$ & $\overline{1,820}$ & $\begin{array}{c}244 \\
-\end{array}$ & $\underline{1}^{145}$ & $\begin{array}{r}95 \\
-\quad 2\end{array}$ & 215 \\
\hline$\$ 24,639$ & $\$ 23,446$ & $\$ 20,673$ & $\$ 20,112$ & $\$ 18,773$ & $\$ 20,915$ \\
\hline$\$ 3,246$ & $\$ 3,460$ & $\$ \quad 543$ & $\$ \quad 859$ & $\$ \quad 859$ & $\$ 715$ \\
\hline $\begin{array}{r}1,659 \\
19,896\end{array}$ & $\begin{array}{r}1,315 \\
18,945\end{array}$ & $\begin{array}{r}1,642 \\
18,955\end{array}$ & $\begin{array}{r}1,743 \\
17,806\end{array}$ & $\begin{array}{r}1,864 \\
16,348\end{array}$ & $\begin{array}{r}1,430 \\
18,984\end{array}$ \\
\hline $\begin{array}{r}\$ 24,801 \\
24,639\end{array}$ & $\begin{array}{r}\$ 23,721 \\
23,446\end{array}$ & $\begin{array}{r}\$ 21,140 \\
20,673\end{array}$ & $\begin{array}{r}\$ 20,408 \\
20,112\end{array}$ & $\begin{array}{r}\$ 19,071 \\
18,773\end{array}$ & $\begin{array}{r}\$ 21,129 \\
20,915\end{array}$ \\
\hline$\$ \quad 162$ & $\$ \quad 275$ & $\$ \quad 467$ & $\$ \quad 296$ & $\$ \quad 298$ & $\$ 214$ \\
\hline
\end{tabular}

Pourcentages de la totalité du passif et de l'actif

\begin{tabular}{rrrrrr}
36.0 & 37.7 & 39.7 & 40.8 & 43.7 & 39.3 \\
55.3 & 50.2 & 59.1 & 58.5 & 55.8 & 59.7 \\
.5 & 4.4 & - & - & - & - \\
\hline 8.2 & $\overline{7.7}$ & \multicolumn{1}{c}{-2} & - & \multicolumn{1}{c}{-5} & 1.0 \\
\hline 100.0 & 100.0 & 100.0 & 100.0 & 100.0 & 100.0 \\
\hline 13.1 & 14.6 & 2.6 & 4.3 & 4.5 & 3.4 \\
6.7 & 5.5 & 7.8 & 8.5 & 9.8 & 6.8 \\
80.2 & 79.9 & 89.6 & 87.2 & 85.7 & 89.8 \\
\hline 100.0 & 100.0 & 100.0 & 100.0 & 100.0 & 100.0 \\
.65 & 1.15 & 2.20 & 1.44 & 1.56 & 1.01 \\
\hline \hline
\end{tabular}

2 Journal de la Chambre de l'Assemblée de l'Ile du Prince-Edouard. (Charlottetown, 1866), Appendice X.

3 Ibid, 1868, Appendice $\mathrm{H}$.

4 Ibid, 1871, Appendice Y.

5 Ibid, 1874, Appendice 0.

6 Ibid, 1875, Appendice Y.

${ }^{7}$ La Gazette du Canada, publiée par ordre (Ottawa, 31 octobre 1885), Vol. XIX, (No 18) : 636 .

8 Ibid, 15 octobre 1887, Vol. XXI, (No 16) : 815 .

9 Ibid, 18 octobre 1890, Vol. XXIV, (No 16) : 696.

10 Ibid, 17 octobre 1891, Vol. XXV, (No 16) : 661. 
les dividendes de la Banque étaient trop élevés; en 1866, le taux de dividende était de 12 pour cent, et, de 1867 à 1875, il a été réduit à 10 pour cent. Il n'existe aucun renseignement couvrant les dix années subséquentes, mais un dividende de 8 pour cent a été payé en 1885, et un dividende de 6 pour cent jusqu'en $1891 .^{33}$ Outre les avantages que leur procurait le fait d'être propriétaires de ce commerce, les actionnaires retiraient un revenu appréciable du capital qu'ils avaient investi.

Au tableau No 1 figurent les bilans de la Banque de Rustico, de 1866 à 1891, la dernière année où un rapport a été déposé auprès du ministère des Finances..$^{34}$ Le bilan du 3 avril 1866 indique l'état de la Banque après une année entière d'opérations. Elle avait progressé rapidement et consenti une totalité de prêts plus considérable que ne l'autorisait la loi - c'est-à-dire deux fois le montant du capital versé. Elle avait alors en circulation des billets dont la valeur dépassait trois fois ce capital. Cette situation a été rectifiée l'année suivante, alors que la Banque a obtenu l'autorisation d'augmenter son capital de façon que la valeur de ses billets et prêts ne dépasse pas la limite légale; et, au cours des années subséquentes, la Banque n'a jamais dépassé cette limite. L'expansion qu'a connue la Banque de 1866 à 1871 se reflète dans les prêts qu'elle a consentis au cours de ces années: $\$ 12,068$ en 1866 jusqu'à $\$ 23,555$ en 1871 . En 1873 et 1875 , il y eut diminution dans la valeur des billets en circulation ainsi que des prêts consentis; c'est cette situation qui a induit

\footnotetext{
Banks of the Dominion of Canada, compilé par N.S. Garland (Ottawa, 1890 , sept. ou oct.). Les rapports de la Banque de Rustico sont insérés parmi les avis légaux, dans la Gazette du Canada, ordinairement dans la livraison de la troisième semaine d'octobre. La Banque de Rustico n'est pas mentionnée dans un grand nombre d'études sur le système bancaire canadien, probablement parce que ses rapports n'apparaissent pas parmi ceux des autres banques à charte. Voir renvois au Tableau No 1 pour la source où ont été obtenus les bilans de cette banque.

Vers la fin du siècle dernier, les livres et archives de la «Farmers' Bank of Rustico » ont été détruits au cours d'un incendie qui consuma la maison où ils étaient conservés. Deux témoins de cet incendie nous ont confirmé ce fait.

33 Appendices au Journal de la Chambre d'Assemblée, 1866-1875; Gazette du Canada, loc. cit., 1885-1891. 3 août 1954 .

${ }^{34}$ Lettre de l'Inspecteur général des banques, ministère des Finances,
} 
l'abbé Belcourt à faire enquête dans les affaires de la Banque et à publier son rapport dans le journal Le Moniteur Acadien. Durant cette période, jusqu'en 1875, les affaires de la Banque ont été administrées de façon prudente; 13 ou 14 pour cent de la totalité de ses ressources étaient en lingots; en 1873 et 1875 , environ 20 pour cent en espèces, lingots ou autres billets de banque. Cette situation lui permettait de racheter facilement ses émissions de billets. Cependant, les réserves étaient minimes: en 1867 , le poste «montant au crédit de la Banque » n'indiquait que $\$ 488$, soit 1.92 pour cent de ses ressources totales; en 1873 , cette somme était réduite à $\$ 162$, soit .65 pour cent des ressources totales. Le dividende de 10 pour cent alors accordé ne permettait pas d'augmenter les réserves. En réalité, on a surtout reproché aux administrateurs de cette Banque de ne pas avoir pourvu à des réserves suffisantes. La situation précaire qu'a connue la Banque durant la plus grande partie de son existence a peut-être empêché que soit adoptée une politique plus réaliste quant à ses réserves.

Après 1875, la Banque a cessé de faire rapport auprès du gouvernement provincial. Et ainsi prenait fin une période des opérations de la Banque. En 1885, lorsqu'elle a de nouveau produit ses états financiers, d'autres personnes en avaient alors assumé la direction: Joseph Gallant en avait été le président, et Adrien Doiron, le caissier, depuis au moins $1880 .{ }^{35}$ Comme son prédécesseur, Adrien Doiron avait été instituteur. Joseph Gallant était cultivateur et commerçant; il était propriétaire d'un magasin situé en face de la Banque.

Les bilans couvrant les années 1885 et 1891 indiquent certains changements; au passif, le capital social accuse une diminution de $\$ 658$; les relations avec la «Union Bank» avaient cessé, car la Banque de la Nouvelle-Ecosse avait acheté cette dernière, et la Banque de Rustico n'acceptait plus de dépôts portant intérêts. Du côté de l'actif, le montant d'or et d'argent que détenait la Banque avait considérablement diminué, et son portefeuille d'effets d'autres banques avait augmenté. Son actif, en

35 Almanach de l'Ile du Prince-Edouard, de Chappelle, pour l'année 1880 (Charlottetown: Theo L. Chappelle, Librairie Diamond). 
lingots et autres billets de banque, représentait, en 1875, 20 pour cent de ses ressources totales, et durant la période de 1885 à 1891, il n'était que de 10 à 14 pour cent. Les billets en circulation de la Banque de Rustico, ainsi que les prêts qu'elle avait consentis, ont baissé durant les années 1885 à 1890 ; la somme des billets en cours a été réduite de $\$ 12,217$, en 1885 , à $\$ 10,466$ en 1890 , et les prêts, pour ces mêmes années, de $\$ 18,955$ à $\$ 16,348$. En 1891, les billets en circulation ont augmenté d'un peu plus de $\$ 2,000$; l'actif en espèces a baissé d'environ $\$ 600$, alors que la totalité des prêts consentis avait augmenté d'au delà de $\$ 2,600$. Durant ces six dernières années, la Banque a continué à payer un dividende de six pour cent. Les années 1880 ont été dures pour les habitants de l'île; les taux d'intérêt étaient probablement plus bas, et il était plus difficile de recouvrer les dettes. Mais en maintenant en circulation un plus grand pourcentage de ses ressources, tel que l'indique la totalité des espèces qu'elle détenait, la Banque a pu-accuser un profit.

La Banque n'était pas tenue de produire un état de profits et pertes, et ainsi nous ne possédons pas de chiffres exacts sur ses opérations. Mais les renseignements contenus dans ses bilans nous permettent d'estimer ses recettes et dépenses. En assumant que, durant la période antérieure à 1875, elle ait exigé un intérêt de 7 pour cent de ses emprunteurs et consenti en moyenne des prêts au montant de $\$ 20,000$, et durant la période postérieure à 1885, un intérêt de 6 pour cent de ses emprunteurs et des prêts d'environ $\$ 17,000$, nous pouvons établir l'état suivant, en nous basant sur les renseignements contenus dans les rapports:

TABLEAU II

FARMERS' BANK OF RUSTICO

Etat reconstruit des recettes et dépenses annuelles moyennes, avant 1875 et après 1885 .

\begin{tabular}{|c|c|c|c|}
\hline Période antérieure à 187 & & Période postérieure à 188 & \\
\hline $\begin{array}{l}\text { Recettes totales, moyenne des } \\
\text { prêts }-\$ 20,000 \text { à } 7 \% \text { d'in- } \\
\text { térêt }\end{array}$ & $\$ 1,400$ & $\begin{array}{l}\text { Recettes totales, moyenne des } \\
\text { prêts }-\$ 17,000 \text { à } 6 \% \text { d'in- } \\
\text { térêt }\end{array}$ & $\$ 1,020$ \\
\hline \multirow[t]{2}{*}{ 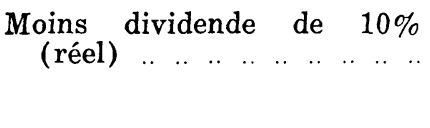 } & 887 & 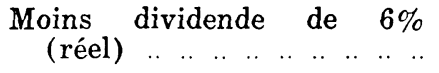 & 493 \\
\hline & 513 & & 527 \\
\hline
\end{tabular}


Sommes estimatives des intérêts non perçus

Sommes disponibles pour dépenses

Moins dépenses estimatives:

Caissier ...

Président et admi-

nistrateurs ........ 100

Autres dépenses ..... 100

Nous ne prétendons pas que les chiffres couvrant les dépenses soient exacts. Il est possible que le caissier ait touché $\$ 300$ par année - ce qui est peu probable, car à cette époque un instituteur dans l'île du Prince-Édouard recevait à peine ce traitement pour une année de travail. Il est possible que les administrateurs n'aient touché aucune rémunération, car dans ce pays on offrait volontiers ses services gratuitement. D'autres dépenses sont indiquées; mais il est difficile d'en établir le montant exact. La Banque ne payait pas de loyer: la salle était à sa disposition, car elle avait été construite à cette fin. Comme elle n'était ouverte qu'une après-midi par semaine, les frais d'éclairage et de chauffage étaient négligeables; le combustible était probablement fourni gratuitement, à même le bois que les paroissiens apportaient pour chauffer l'église. La papeterie, les fournitures et l'impression des billets constituaient les seules dépenses possibles. Le montant des dettes non recouvrables était peut-être inférieur ou supérieur à celui qui est indiqué. Le montant à l'actif de la Banque - c'est-à-dire la différence entre le passif et l'actif — n'a guère varié au cours de cette époque.

$\mathrm{Au}$ bas mot, ce n'est guère un chiffre d'affaires important. La totalité de son capital, auraient à peine suffi à alimenter la caisse d'une banque à charte dans l'une de nos villes importantes. On comprend facilement pourquoi les économistes qui ont retracé l'historique de l'industrie bancaire au Canada, ont ignoré la «Farmers' Bank of Rustico ». Evidemment, il ne faut pas appliquer à cette Banque les standards d'aujourd'hui. A cette époque, 
un prêt de $\$ 50$ constituait une transaction importante. Nous avons pu apprendre que le montant minimum des prêts consentis par cette Banque était de $\$ 35$.

Ce sont là des opérations peu importantes; mais elles étaient effectuées en toute conscience et honnêteté. La Banque annonçait qu'elle était ouverte tous les mercredis après-midi. ${ }^{36}$ Ceux qui se rappellent cette époque racontent que chaque semaine administrateurs et clients venaient à la Banque à dos de cheval, ces premiers pour y tenir leur assemblée régulière. On peut aussi signaler qu'au moins durant les dernières années de son existence, la Banque desservait non seulement la communauté acadienne, mais aussi la population de langue anglaise. Les habitants de descendance acadienne et anglo-saxonne prédominent dans la région de Rustico, bien qu'on y trouve aussi des citoyens d'origine irlandaise et écossaise. Certains administrateurs et actionnaires de la Banque étaient de langue anglaise, car c'était une banque communautaire à laquelle s'adressaient également des Acadiens d'autres paroisses. Dans les deux autres centres acadiens, des agents s'occupaient des formalités relatives aux prêts et des garanties des emprunteurs, qui, cependant, devaient se rendre à Rustico en personne afin d'y signer le billet et toucher l'argent. A Egmont Bay, quelque 50 milles de Rustico, l'agent était J.-O. Arsenault, député à la législature, de 1867 à 1895, alors qu'il a été nommé sénateur. L'agent de Tignish, à l'autre extrémité de l'île, à 80 milles de Rustico, était Stanislas-F. Poirier qui fut député à la Chambre des Communes pendant plusieurs années. (Signalons qu'au Canada les députés fédéraux voyagent gratuitement sur les chemins de fer; mais la Banque devait surveiller les dépenses). La Banque était petite, mais elle subvenait aux besoins de petits cultivateurs. Et même aujourd'hui, les résidents de Rustico qui se rappellent cette banque estiment hautement l'œuvre qu'elle a accomplie. Dans cette institution, nous retrouvons, dans toute son application, le caractère de modestie et d'intimité des banques du peuple. ${ }^{37}$

36 Ibid.

37 Nous avons recueilli les faits rapportés dans ce paragraphe au cours de l'été 1954 , lors de plusieurs interviews avec de vieux résidents de l'Ile du Prince-Edouard. 
La Banque de Rustico avait, originalement, obtenu une charte d'une durée de 20 ans, laquelle devait expirer le $1^{\text {er }}$ juin 1883. ${ }^{38}$ Comme elle ne pouvait se conformer aux conditions exigées par la législation fédérale, la Banque, apparemment, n'avait d'autre recours que de vendre son actif à une institution plus importante; c'est ce qu'alors devaient faire les petites banques canadiennes.

Une année avant l'expiration de sa charte, la Banque de Rustico avait demandé au parlement fédéral de la renouveler; mais, en 1882, aucune décision n'avait été rendue à cet égard. Dans les coulisses, certaines pressions s'exerçaient à l'encontre de la Banque. ${ }^{39}$ Le 22 février, un bill destiné à prolonger la durée de la constitution en corporation de la «Farmers' Bank of Rustico » a été déposé à la Chambre des Communes; le lendemain, deuxième lecture a été donnée à ce bill. Il y eut retard au Sénat où une pétition relative à ce même bill avait été déposée le 15 février. Le 9 avril, la Chambre des Communes adoptait ce bill. ${ }^{40}$ Première lecture a eu lieu au Sénat le 12 avril, mais la deuxième lecture en a été ajournée au 16 avril. ${ }^{41}$ Le 18 avril, lors du débat qu'a tenu le Sénat à l'occasion de cette deuxième lecture, l'honorable sénateur Haythorne a parlé de «la forte opposition manifestée, durant ces deux ou trois derniers jours, contre les bills concernant les banques »; il a déploré qu'il fût même nécessaire de discuter la présente loi. Cependant, il a exposé, en termes sympathiques, l'historique de la Banque de Rustico, il a mentionné son chiffre d'affaires minime; et ensuite il a expliqué les dispositions de la Loi. Le premier article du bill prolongeait de neuf années la durée de la charte, soit jusqu'au $1^{\text {er }}$ juillet 1891. En vertu de l'article 2, les rapports qui antérieurement étaient adressés aux autorités provinciales, devaient doréart. 44.

38 «Loi constituant en corporation la Farmers' Bank of Rustico»,

39 Débats du Sénat du Dominion du Canada, 1883, Première Session, Cinquième Parlement (Ottawa; Rapportés, édités et publiés par Holland Bros., sténographes officiels du Sénat du Canada, 1883), 271.

40 Débats de la Chambre des Communes du Dominion du Canada, Première Session, Cinquième Parlement (Ottawa, 1883), 67, 77 et 501.

41 Journaux du Sénat du Canada, Première Session du Cinquième Parlement (Ottawa, 1883), XVII: 39, 48, 143, 150. 
navant l'être au ministre des Finances fédéral. Ces règlements s'appliquaient aux banques canadiennes, sauf à celles qui avaient été constituées en corporation, antérieurement, par les législatures. Le troisième et plus important article du bill exigeait que la Banque réduise son émission de billets; elle devait retirer chaque année $121 / 2$ pour cent des billets dépassant le montant de son capital social, de sorte que, en 1891, la valeur totale de ces billets en circulation ne dépasse pas le capital versé de la Banque. Un article de ce bill stipulait que le Parlement se réservait le droit d'examiner ledit bill de nouveau à l'expiration de cette période de neuf années. ${ }^{42}$ Le comité a retranché cette disposition, et le premier article du bill, tel qu'adopté, stipulait que la charte expirait automatiquement le $1^{\mathrm{er}}$ juillet 1891 . Le Sénat a adopté ce bill le 24 avril, la Chambre des Communes, le 30 avril, et il a été sanctionné le 25 mai $1883 .^{43}$

\section{IV}

Quelle était l'attitude des banques à charte à l'égard de cette banque peu importante, inoffensive, mais provocante, et qui refusait de cesser ses opérations ? Au début de son existence, la Banque de Rustico, tel que l'indique le Tableau No 1, devait certainement bénéficier d'une entente avec la «Union Bank ». Les bilans semblent indiquer que la «Union Bank», établie à Charlottetown, agissait comme agent de compensation pour la Banque de Rustico, et qu'elle n'avait qu'un faible solde débiteur à percevoir. Et ainsi, le caissier de la Banque de Rustico n'était pas tenu de transporter de l'or au cours de ses voyages hebdomadaires à Charlottetown; en conséquence, il compensait le montant des billets des banques de Charlottetown contre celui de la Banque de Rustico - ce qui constituait un arrangement commode. Cependant, à la fin des années 1880, les bilans n'indiquent

42 Débats du Sénat, op. cit., 271-273..

43 Journaux du Sénat, op. cit., 158, 175, 176, 196 et 295; Actes du Parlement du Dominion du Canada, passés en la quarante-sixième année du Règne de $\mathrm{Sa}$ Majesté la Reine Victoria, et en la Première Session du Cinquième Parlement (Ottawa, 1883), II (Lois locales et privées) : 14-15, chap. 49 . 
pas que ces ententes subsistaient. Etant donné que la charte de la Banque de Rustico devait expirer en 1891, il n'est pas étonnant que les banques de Charlottetown aient hésité à accepter les billets de la Banque de Rustico. Il est curieux de constater que la Banque de Rustico n'aurait subi aucun tort si les autres banques eussent refusé de racheter ses billets; en agissant ainsi, ces banques auraient maintenu en circulation les billets de la Banque de Rustico. C'est là l'application de la Loi de Gresham. Etant donné l'insuffisance de monnaie durant les dernières années du dix-neuvième siècle, il devenait facile de mettre en circulation des billets de banque ayant cours légal. ${ }^{44}$

On peut se renseigner davantage sur l'attitude des banques à charte à l'égard de la Banque de Rustico en lisant les propos tenus par deux députés fédéraux de l'île du Prince-Édouard, M. Hackett, du district de Prince, et M. Davies, du district de Queens, lors d'un débat à la Chambre des Communes en 1886. On discutait un bill présenté par M. Orton et visant à établir des banques de cultivateurs. (Le sujet de ce bill n'entre pas dans la présente étude). M. Hackett approuvait les banques de cultivateurs, et après avoir traité des difficultés qu'éprouvent les cultivateurs qui désirent obtenir crédit, voici ce qu'il dit concernant la Banque de Rustico:

Je sais personnellement que l'établissement de petites banques constitue une affaire vitale aux inté-

44 A cette époque, des bars ouverts existaient dans l'Ile du PrinceEdouard. On y vendait le rhum 3 cents le verre. Une méthode courante de mettre en circulation un billet de la «Farmers' Bank 》 consistait à s'en servir pour se payer un coup après le long et dur trajet de Rustico. On remettait au cultivateur des pièces de monnaie, qui étaient acceptées partout. Il appartenait alors à l'hôtelier de faire circuler le billet. (Ceci m'a été raconté par un ancien inspecteur d'écoles qui, dans sa jeunesse, a habité avec des parents un des hôtels de Charlottetown. J.T.C.)

Cette pratique explique peut-être le sort d'un grand nombre de billets de la Banque de Rustico; l'Ile du Prince-Edouard bénéficiant à cette époque d'un commerce maritime considérable, voyageurs et marins emportaient avec eux ces billets qui s'égaraient ou ne revenaient jamais à Rustico. Naturellement, un certain nombre ont été perdus ou détruits accidentellement - ce qui confirme les assertions de Ross. Les billets de la Banque de Rustico, en coupures de $\$ 1$ et $\$ 5$, portaient, sur un côté seulement, de magnifiques reproductions de scènes rurales. Ce travail était exécuté par une firme de New-York. On en trouve dans maintes collections. Howard, op. cit., 42, reproduit un fac-similé d'un billet de $\$ 1$. 
rêts des cultivateurs de ce pays. Il y a quelque vingt ans, un missionnaire catholique romain dévoué, l'abbé Bellecourt (sic), curé d'une paroisse de l'Ile du Prince-Edouard, voyant que la population de cette localité se trouvait dans une situation difficile du fait qu'elle devait s'adresser à des usuriers afin d'emprunter de faibles sommes d'argent, établit une petite banque appelée la «Farmers' Bank of Rustico »... Cette Banque se composait exclusivement de cultivateurs; les administrateurs étaient tous des cultivateurs; le gérant était également un cultivateur; cette institution pratique ses affaires depuis vingt ans, en dépit de toutes les autres banques du pays qui s'efforcent de la faire disparaître, d'empêcher que ses billets aient cours et qu'elle puisse exercer ses opérations en général. Mais nonobstant cette opposition, la Banque était administrée selon des principes d'affaires solides; elle existe depuis vingt ans; ses opérations ont été très florissantes, et l'on regrette profondément, dans cette région, qu'elle soit obligée de liquider ses affaires. ${ }^{45}$

M. Davies a admis le bien-fondé d'une partie des remarques de M. Hackett, mais il a nié que les autres banques aient été hostiles à la Banque de Rustico; voici ce qu'il a dit:

Les faits que l'hon. député rapporte concernant la formation de la Banque sont parfaitement vrais; il est également vrai que la population a grandement bénéficié des opérations que cette banque a exercées dans cette région. Mais lorsque mon honorable ami prétend que les autres banques se méfient de cette institution et ont tenté de la faire disparaître, je lui signalerai que, durant les dix ou douze dernières années, j'ai été administrateur d'une de ces banques, et je puis l'assurer que, bien au contraire, ces dernières se réjouissaient de voir la Banque de Rustico prospérer; elles souhaitent que la durée de sa charte soit prolongée, car elles savent que la population de cette région bénéficie grandement de ses opérations. ${ }^{46}$

45 Rapport officiel des Débats de la Chambre des Communes du Dominion du Canada, Quatrième Session, Cinquième Parlement. 49 Victoria, 1886 (Ottawa, 1886), XXI: 584.

46 Idem. 
A ceci, M. Hackett a répondu :

Il est possible que mon honorable ami ait raison en ce qui concerne l'attitude de la banque dont il est l'un des administrateurs; mais il y a environ cinq ans, une des principales banques de Charlottetown a refusé quelques billets de la Banque de Rustico dont j'étais porteur, et ce, à mon avis, en vue de faire disparaître cette petite banque. ${ }^{47}$

Ainsi, nous avons deux opinions opposées; toutes deux contiennent probablement une part de vérité. Dans les régions où les banques locales étaient possédées par la population de l'endroit et administrées par des personnes connaissant la dure vie des cultivateurs de l'île, on appréciait avec bienveillance l'œuvre de la Banque de Rustico. Mais l'existence d'une institution aussi peu importante devait nécessairement ennuyer les banques puissantes qui venaient s'établir dans la localité. Cette institution n'avait plus sa raison d'être dans le nouveau système économique. La nouvelle époque établissait le principe de la centralisation; l'existence des petites banques locales - pour ne pas parler de la Banque de Rustico - était chose du passé.

Mais le coup de mort n'avait pas encore été donné à la Banque de Rustico. La prolongation de sa charte devait prendre fin le $1^{\text {er }}$ juillet 1891 . Le 13 mai 1891 , la pétition suivante était inscrite aux Journaux de la Chambre:

De Peter McIntyre, évêque de Charlottetown, et d'autres personnes de l'Ile du Prince-Edouard, demandant l'adoption d'une loi qui prolonge la validité de la Loi constituant en corporation la «Farmers' Bank of Rustico $\gg .48$

Il fallait se hâter. Le 15 mai, le bill visant à prolonger la charte était déposé et lu la première fois; le 18 mai, il recevait deuxième lecture et était renvoyé au Comité des banques et du commerce. Le Comité en a fait rapport le 22 juin; le bill a immédiatement été inscrit à l'Ordre du jour, adopté le 24 juin, et transmis au Sénat. ${ }^{49}$

47 Idem.

48 Journaux de la Chambre des Communes du Dominion du Canada, Première Session du Septième Parlement du Canada (Ottawa, 1891), XXV: 84.

49 Ibid., 110, 111, 126, 229, 233, 245, 246. 
Ce bill a reçu première lecture au Sénat le 26 juin, qui était la dernière journée de séance avant le congé de la fête du Dominion. Un bill semblable concernant la «Albion Mines Savings Bank », une petite institution de la Nouvelle-Écosse, était également à l'étude. L'hon. M. Abbott a franchement admis l'objet du bill:

Ces bills renfermaient des dispositions ... destinées à faire disparaître ces deux petites banques. La Banque de Rustico avait le droit d'émettre des billets, et ce bill lui en enlevait le droit graduellement jusqu'à ce que, éventuellement, la totalité de ses billets ait été retirée de la circulation; et dès lors, la Banque pourra se fusionner avec une autre institution, ou lui transporter son actif.

Les règles ayant été suspendues, le bill a passé par toutes les étapes réglementaires, il a reçu troisième lecture et il a été adopté. ${ }^{50}$

Alors la «Farmers' Bank of Rustico» connut ses derniers jours de gloire. Afin de satisfaire aux délais, le Gouverneur général, ce jour même, a dû se rendre au Parlement spécialement pour sanctionner ce bill. C'était la dernière journée possible; immédiatement après que le Gouverneur eût sanctionné ce bill, les deux Chambres se sont ajournées au 2 juillet. ${ }^{51}$

Alphonse Desjardins, le pionnier des caisses populaires de Québec et de la «American Credit Union», est venu au Parlement du Canada, en 1892, en qualité de reporter au Hansard. ${ }^{52}$ En lisant les débats parlementaires, il s'est familiarisé avec la «Farmers' Bank of Rustico ». Il en a probablement causé avec les députés de l'île du Prince-Édouard. De toute façon, il était renseigné sur cette Banque qui a exercé une certaine influence

50 Débats du Sénat du Dominion du Canada (Ottawa, 1891), 144.

51 Journaux du Sénat du Canada, Première Session du Septième Parlement du Canada (Ottawa, 1891), XXV: 153.

52 Cyrille Vaillancourt et Albert Faucher, Alphonse Desjardins, pionnier de la coopération d'épargne et de crédit en Amérique (Lévis: Le Quotidien, Ltée, 1950), 26. 
sur les projets qu'il devait réaliser. ${ }^{53}$ En étudiant les épreuves qu'avait subies cette Banque, Desjardins s'était sans doute rendu compte des obstacles d'ordre légal et constitutionnel qu'il lui faudrait affronter s'il désirait établir des banques du peuple au Canada. Plus tard, après une étude approfondie de ce sujet, et une correspondance prolongée avec des autorités européennes, il élabora un projet de banques du peuple fondé sur le modèle Luzzatti. La «Farmers' Bank of Rustico » peut être considérée comme précurseur des caisses populaires et du mouvement des «credit unions » qui couvrent maintenant l'Amérique du Nord.

Il est évident que la loi de 1891, intitulée: "Acte concernant la Farmers' Bank of Rustico ${ }^{54}$ a été rédigée à la hâte ou de façon négligente. Elle avait pour objet de faciliter la liquidation de cette Banque; mais les dispositions en étaient tellement rigides et si peu pratiques qu'il était impossible de les appliquer. La Banque de Rustico a obtenu que sa vie soit prolongée de trois années, jusqu'au $1^{\text {er }}$ juillet 1894 . Dix pour cent des billets en circulation au $1^{\text {er }}$ juillet 1891 devaient être retirés à la fin de la première année; vingt pour cent à la fin de la deuxième année, et le reste, à la fin de la troisième année. La Banque était autorisée à se fusionner avec n'importe quelle compagnie de prêts, ou à lui vendre son actif, à condition qu'une telle transaction ait été approuvée par la majorité des actionnaires, représentant au moins les deux tiers du capital. En vertu de cette loi, la compagnie de prêts qui acquérait la Banque assumait la responsabilité de toutes les dettes et de tous les engagements de ladite banque, ainsi que de toutes les poursuites en justice pouvant être intentées à l'égard de ces dettes ou engagements. Le dernier article de la loi (article 6) stipulait:

6. Avant que cette fusion ne soit mise à effet ou n'entre en vigueur, tous les billets de la Banque en circulation présentés au rachat seront remboursés, et une somme prise sur l'actif de la Banque égale

53 Ibid, 27; également: C. Vaillancourt, «Credit Unions in Quebec 》, The Bridge, VI: No 1, (avril 1941) 76.

${ }_{54}$ Actes du Parlement du Dominion du Canada, passés en la session tenue en les cinquante-quatrième et cinquante-cinquième années du règne de Sa Majesté la Reine Victoria, en la Première Session du Septième Parlement. (Ottawa, 1891), Vol. 2 (Lois locales et privées) : 168-169, chap. 113. 
au montant restant alors des billets destinés à la circulation émis par la Banque et non rachetés, sera déposée entre les mains du ministre des Finances et Receveur général; la somme ainsi déposée sera gardée par le ministre des Finances et Receveur général et employée au rachat, sur présentation (dans les trois ans qui suivront ce dépôt), des billets restant ainsi en circulation, sans intérêt, et toute balance non ainsi appliquée sera remise, à l'expiration de cette période, à ladite compagnie de prêt.

La disposition de la Loi de 1883 relative à la réduction du montant de billets en circulation accordait à la Banque un délai de 9 années pour réduire ces billets au total de son capital versé; cette réduction représentait une somme annuelle d'environ $\$ 500$, soit un total d'environ $\$ 4,000$. La Banque a ignoré cette disposition jusqu'en 1887, alors qu'elle a commencé à effectuer cette réduction. Probablement, le ministre des Finances insistait pour que la Banque obéisse aux stipulations de la Loi. Au mois d'octobre 1890, la valeur des billets en circulation ne dépassait que de $\$ 2,000$ le montant du capital versé, tel que l'indique le Tableau No 1. En consultant le bilan du 7 octobre 1890 de la Banque de Rustico, on peut estimer qu'au moment où la loi de 1891 a été adoptée, cette institution pouvait en toute sécurité liquider ses affaires. La totalité de son passif consistait en billets d'une valeur de $\$ 10,466$. En regard, elle avait à son actif $\$ 2,723$ en espèces, et des prêts à recouvrer d'une valeur de $\$ 16,348$. En soustrayant la somme en caisse de la valeur des billets en circulation, le solde de $\$ 7,743$ représentait moins que la moitié de la totalité des prêts à recouvrer. En d'autres termes, si seulement 50 pour cent des prêts pouvaient être recouvrés, il devenait possible de racheter la totalité des billets. Probablement, dans une large mesure, emprunteurs et actionnaires étaient les mêmes personnes; en appliquant au capital social la moitié des prêts recouvrables, et en mettant à part le solde aux fins de racheter les billets en circulation, il eut été possible d'effectuer la liquidation sans accuser de perte. En outre, un grand nombre de billets avaient été perdus; si l'on avait exigé que les demandes de remboursement aient été déposées dans un délai déterminé — disons trois 
ans - il en serait probablement résulté un surplus à répartir entre les actionnaires.

La Loi de 1891 stipulait que ce surplus devait être versé à la compagnie de prêts qui achèterait la Banque. En outre, la compagnie de prêts acquéreuse pouvait établir les termes auxquels les emprunteurs de la Banque devaient rembourser leurs emprunts à cette compagnie de prêts. Il semble que la Banque de Rustico n'ait guère été sévère lorsqu'il s'agissait de recouvrer les emprunts qu'elle avait consentis à ses propres membres. ${ }^{55}$

Quelle a été la réaction des membres ? La dernière assemblée qu'a annoncée la Banque a été tenue le 2 juillet $1891 .^{56} \mathrm{Il}$ serait intéressant de pouvoir consulter le compte rendu de cette assemblée. Mais le bilan du 6 octobre 1891 - le dernier qu'ait produit la Banque - indique qu'au lieu de restreindre les opérations, les administrateurs ont augmenté le montant des prêts de quelque $\$ 2,600$. Pour ce faire, ils ont réduit de $\$ 578$ le montant en caisse, et augmenté de $\$ 2,022$ la somme de leurs billets. Et ensuite, d'après ce que l'on peut constater, ils n'ont rien fait. Apparemment, la Gazette Royale n'a publié aucun autre avis concernant cette affaire; mais il est encore plus surprenant de constater qu'aucun résident de l'Île du Prince-Édouard que nous avons interviewé à cet égard ne semble savoir ce qui s'est produit. Dans un petit pays comme l'île du Prince-Édouard, s'il était survenu quelque chose, quelques-uns parmi les avocats de l'époque ou parmi les plus vieux résidents en auraient certainement eu connaissance. Il est vrai que certaines personnes parlent de la «faillite» de la Banque, tout comme certaines études

55 Nous avons fouillé les procès-verbaux de la Cour suprême de l'Ile du Prince-Edouard, couvrant la plupart des années 1890. C'est là qu'eussent apparu pareilles causes en recouvrement de dettes. Nous n'avons rien trouvé concernant la « Farmers' Bank of Rustico», alors que nous y avons relevé plusieurs causes de la Banque de Nouvelle-Ecosse.

Un ancien juge de la Cour suprême, maintenant retraité et qui a commencé, encore jeune, à étudier le droit immédiatement après que la Banque de Rustico eût cessé ses opérations, nous a dit, au cours d'une interview, qu'il ne se rappelait aucune poursuite en justice relative à la «Farmers' Bank of Rustico».

56 Gazette Royale, publiée par ordre (Charlottetown, Ile du PrinceEdouard, numéro du 20 juin 1891), XVIII: (No 25) 105. La Banque de Rustico annonçait toutes ses assemblées dans ce journal officiel. 
sur les institutions bancaires du Canada mentionnent en passant la «faillite » ou la « liquidation » de la Banque de Rustico; mais il semble qu'il n'existe aucune mention officielle indiquant que cette Banque ait fait faillite ou qu'elle ait été liquidée. Apparemment, elle a tout simplement « cessé d'exister ».

On peut se demander comment une banque comme celle de Rustico pourrait tout simplement cesser ses opérations sans que rien ne s'ensuive. La chose est possible. Aux États-Unis, plusieurs 《Credit Unions» qui obtiennent leur charte des divers États, ont sans doute connu le même sort, surtout lorsque les départements contrôlant les opérations des banques ne s'intéressent guère attentivement ou sérieusement aux affaires de ces institutions. Sauf dans quelques États qui possèdent des départements bancaires éveillés, on chercherait en vain des statistiques concernant la liquidation de «Credit Unions » possédant des chartes d'État. Aucune surveillance n'était exercée sur les opérations de la Banque de Rustico; on lui demandait simplement de déposer un rapport annuel. En réalité, la Banque ne connaissait que peu de restrictions, sauf qu'un actionnaire ou un créancier pouvait lui intenter une poursuite en justice. Il était peu probable qu'un actionnaire qui lui avait emprunté de l'argent la poursuive. Si l'on présume que la plupart des billets en circulation avaient été emportés hors de l'île ou détruits accidentellement ce qui n'est pas incompatible avec la pratique courante de cette époque - il n'était guère probable qu'un porteur de billets intente une action en justice contre cette Banque. Au 6 octobre 1891, comme l'indique le Tableau No 1, la Banque avait en caisse la somme de $\$ 2,145$ aux fins de rembourser ses billets, advenant qu'un détenteur ait pris la peine de se rendre à Rustico pour en exiger le paiement. Il semble possible, et même probable, que la Banque ait simplement cessé d'exister.

Dans cette affaire, il faut tenir compte d'un autre facteur: il semble que le caissier, Adrien Doiron, ait souffert d'une dépression nerveuse vers cette époque. Une personne qui l'a bien connu se rappelle que ces difficultés l'avaient grandement affecté. Il ne semblait pas comprendre pourquoi les autres banques ne voulaient plus accepter les billets de la Banque de Rustico; il sem- 
blait moralement déprimé. ${ }^{57}$ Etant donné l'état d'Adrien Doiron, il est facile de comprendre que la Banque soit allée à la dérive. Sa charte expirait le $1^{\text {er }}$ juillet 1894. Adrien Doiron mourut quelques jours plus tard, à l'âge de 55 ans; le 18 juillet 1894, il était inhumé au cimetière de la paroisse, à l'ombre de la Banque qu'il avait tant aimée. ${ }^{58}$

"La mort confirme que la vie existe; elle n'en constitue pas une dénégation. »La Banque de Rustico, comme son caissier, est disparue. Outre les services qu'elle a rendus à la population de la région, il est possible que cette Banque ait exercé son influence la plus importante en inspirant le mouvement des sociétés coopératives de crédit d'Alphonse Desjardins. En étudiant les opérations de la «Farmers' Bank of Rustico», Desjardins a dû se rendre compte que la formule des banques commerciales ne pouvait s'appliquer aux banques du peuple. Il a sans doute constaté que des obstacles d'ordre financier et constitutionnel nuisaient à l'établissement de banques du peuple au Canada. Il a résolu les problèmes inhérents à la formule et aux techniques bancaires, et il a réussi à contourner les obstacles d'ordre constitutionnel.

Cependant, à certains égards, il semblait que la Banque de Rustico violait les principes des banques du peuple, tout comme elle avait bravé la politique bancaire du gouvernement du Canada ainsi que les grandes banques commerciales. Comme les banques du peuple ne sont ordinairement que de petites institutions exerçant leurs affaires durant une partie de la semaine, elles doivent se grouper sous forme d'un mouvement organisé afin d'obtenir protection, renseignements et inspiration. L'abbé Belcourt s'en est rendu compte. La réalisation la plus remarquable de la Banque de Rustico réside probablement dans le fait que, durant 30 années, elle ait pu continuer à pratiquer ses opérations comme institution indépendante et isolée. Une agence disciplinaire centrale doit également vérifier les comptes des banques $\mathrm{du}$ peuple et corriger les abus qui peuvent se glisser dans leurs

57 Interview avec Moïse Gallant, instituteur à la retraite et un parent d'Adrien Doiron.

58 Renseignements puisés dans les archives de la paroisse de SaintAugustin, Rustico, Ile du Prince-Edouard, et certifiés par l'abbé J. Clarence Pitre. 
opérations; encore une fois, la Banque de Rustico a eu la bonne fortune d'obtenir les services de fonctionnaires et d'administrateurs honnêtes et dévoués. Ce n'est pas la Loi des banques qui assure une bonne gestion; mais une loi qui stipule que des réserves suffisantes doivent être mises à part et qui indique la procédure à suivre, peut également inspirer à la gérance les mesures qu'elle doit adopter. Il est extraordinaire de constater que la Banque de Rustico ait pu exercer ses opérations sans posséder de réserves, et n'observer que les règlements qu'elle s'imposait ellemême; voilà un hommage à la prudence et à l'honnêteté de la population de cette région.

En définitive, la « Farmers' Bank of Rustico » constitue un excellent exemple d'une institution qui a su se développer afin de satisfaire les besoins d'une population. La Banque de Rustico, comme institution sociale, n'est réellement pas morte; son œuvre subsiste toujours. Aujourd'hui, dans cet immeuble de pierres rouges, une caisse populaire moderne qu'administrent les cultivateurs de Rustico, poursuit l'œuvre de la première banque du peuple.

John T. CroteaU, D.Ph., Professeur d'économie,

Université de Notre-Dame, Notre-Dame, Etat d'Indiana, E.-U. 